\title{
Advertising and irreversible opinion spreading in complex social networks
}

\author{
Julián Candia \\ Center for Complex Network Research and Department of Physics, \\ Northeastern University, Boston, MA 02115, USA \\ INIFTA, CCT La Plata, Universidad Nacional de La Plata, \\ La Plata, Argentina \\ Email address: jcandia@nd.edu
}

October 29, 2018

\begin{abstract}
Irreversible opinion spreading phenomena are studied on smallworld and scale-free networks by means of the magnetic Eden model, a nonequilibrium kinetic model for the growth of binary mixtures in contact with a thermal bath. In this model, the opinion of an individual is affected by those of their acquaintances, but opinion changes (analogous to spin flips in an Ising-like model) are not allowed. We focus on the influence of advertising, which is represented by external magnetic fields. The interplay and competition between temperature and fields lead to order-disorder transitions, which are found to also depend on the link density and the topology of the complex network substrate. The effects of advertising campaigns with variable duration, as well as the best cost-effective strategies to achieve consensus within different scenarios, are also discussed.
\end{abstract}

Keywords: Complex networks; sociophysics; kinetic growth models; phase transitions; nonequilibrium processes.

\section{Introduction}

Many interdisciplinary fields of science are nowadays benefitting from valuable tools, insight and procedures borrowed from long-standing, traditional 
applications of statistical physics [1, 2, 3, 4, 5]. In particular, a rich variety of social phenomena, such as social influence and self-organization, cooperation, opinion formation and spreading, have recently been studied using models and methods of statistical physics (see e.g. [6, 17, 8, 9, 10, 11, 12, 13, 14, 15, 16, 17, 18, 19, 20, 21]). In some of these investigations, social processes were studied by means of Ising-type spin models [22, 23, 24, 25, 26, 27, 28, 29], in which spin states denote different opinions or preferences, while the coupling constant describes the convincing power between interacting individuals in competition with the "free will" given by the thermal noise.

Four decades ago, seminal observations by Milgram [30] showed that the mean social distance between a pair of randomly selected individuals was astonishingly short, typically of a few degrees. Since then, the phenomenon of average shortest path-lengths that scale logarithmically with the system size, known as small-world effect, has been found ubiquitously in many different real networks, which also generally show highly interconnected local neighborhoods.

The well-studied classical random graphs, which are networks built by linking nodes at random, display the small-world effect but have much lower connectivities than those usually observed in real networks. To address this problem, the so-called small-world networks were proposed few years ago [31, 32 as a realization of complex networks having short mean path-lengths (and hence showing the small-world effect) as well as large connectivities. Starting from a regular lattice, a small-world network is built by randomly adding or rewiring a fraction $p$ of the initial number of links. Even a small fraction of added or rewired links provides the shortcuts needed to produce the smallworld effect, thus displaying a global behavior close to that of a random graph, while preserving locally the ordered, highly connected structure of a regular lattice.

Small-world networks, showing the appropriate topological features observed in real social networks, can thus be meaningfully used as spin model substrates in order to investigate different social phenomena. In this context, irreversible opinion spreading phenomena on small-world [33, 34] and other complex networks 35] have been studied by means of the so-called magnetic Eden model (MEM), a nonequilibrium kinetic growth model in which the deposited particles have an intrinsic spin and grow in contact with a thermal bath [36, 37, 38] (for a review, see Ref. [39]). The MEM defined on smallworld networks present continuous order-disorder phase transitions taking place at finite critical temperatures for any value of the rewiring probability $p>0$ [33], a phenomenon analogous to observations reported previously in the investigation of equilibrium spin systems [23, 24].

According to the growth rules of the MEM, which are given in the next 
Section, the opinion or decision of an individual are affected by those of their acquaintances, but opinion changes (analogous to spin flips in the Ising model) do not occur. It should be noticed that this behavior contrasts with other models of opinion formation and evolution, which generally consider the possibility of changing opinion. As such, the MEM is applicable to scenarios were opinion thermalization processes are unlikely to occur.

Within this context, the aim of this work is to focus on the influence of advertising, which is represented by an external, homogeneous magnetic field. The interplay and competition between temperature and fields lead to orderdisorder transitions, which are investigated by means of order-parameter probability distributions and ensemble averages. The corresponding temperature vs. magnetic field order-disorder phase diagram is obtained, and the role played by shortcuts is discussed. Moreover, the effects of advertising campaigns with variable duration, as well as the best cost-effective strategies to achieve consensus within different scenarios, are also investigated.

Furthermore, in order to explore effects arising from the nature of the complex network substrate, we examine the MEM growing on scale-free networks. The degree distribution of these networks, $P(k)$, which measures the probability that any randomly chosen node has $k$ links, follows a power-law, i.e. $P(k) \sim k^{-\gamma}$. Unlike the Poisson degree distribution of classical random graphs and small-world networks, power-law distributions lack any characteristic scales. The structure of scale-free networks provides an interesting setting in which social roles differ significantly among individuals. Indeed, the most connected nodes in the scale-free network (usually designated as hubs) represent highly influential social leaders, whose opinions can potentially affect the decisions of many other individuals in the society.

This paper is organized as follows: in Section 2, details on the model definition and the simulation method are given; Section 3 is devoted to the presentation and discussion of the results, and Section 4 contains the conclusions.

\section{Model and simulation method}

In this work, we consider two paradigmatic types of complex network model: (a) the one-dimensional, nearest-neighbor, adding-type small-world network (SWN) [40, 41], and (b) the Barabási-Albert (BA) scale-free network (SF) [42, 43, 44].

In case (a), starting with a ring of $N$ sites and $N$ bonds, a SWN realization is built by adding new links connecting pairs of randomly chosen sites. For each bond in the original lattice, a shortcut is added with probability 
p. During this process, multiple connections between any pair of sites are avoided, as well as connections of a site to itself. Since the original lattice bonds are not rewired, the resulting network remains always connected in a single component. On average, $p N$ shortcuts are added and the mean coordination number is $\langle z\rangle=2(1+p)$.

In case (b), SF networks are built by following the preferential attachment growth mechanism: starting with a small fully-connected graph of size $m$, a new node with $m$ edges is added at every time step and is connected to $m$ different nodes already present in the system, where the probability for an already existing node to acquire a new link is proportional to its degree. The BA type of scale-free network is characterized by power-law degree distributions, i.e. $P(k) \sim k^{-\gamma}$, where the degree exponent is $\gamma=3$.

Once the network is created, a randomly chosen up or down spin is deposited on a random site. Then, growth takes place by adding, one by one, further spins to the immediate neighborhood (the perimeter) of the growing cluster, while taking into account the corresponding interaction energies. By analogy to the Ising model, the energy $E$ of a configuration of spins is given by

$$
E=-J \sum_{\langle i j\rangle} S_{i} S_{j}-H \sum_{i} S_{i}
$$

where $S_{i}= \pm 1$ indicates the orientation of the spin for each occupied site (labeled by the subindex $i$ ), $J>0$ is the ferromagnetic coupling constant between nearest-neighbor (NN) spins, $H>0$ is the external field, and $\langle i j\rangle$ indicates that the summation is taken over all pairs of occupied NN sites. As with other spin systems defined on complex networks, the magnetic interaction between any pair of spins is only present when a network link (either a lattice bond or a shortcut) connects their sites.

Setting the Boltzmann constant equal to unity $\left(k_{B} \equiv 1\right)$, the probability for a new spin to be added to the (already grown) cluster is defined as proportional to the Boltzmann factor $\exp (-\Delta E / T)$, where $\Delta E$ is the total energy change involved and $T$ is the absolute temperature of the thermal bath. Energy, magnetic field and temperature are measured in units of the NN coupling constant, $J$, throughout. At each step, all perimeter sites have to be considered and the probabilities of adding a new (either up or down) spin to each site must be evaluated. Using the Monte Carlo simulation method, all growth probabilities are first computed and normalized, and then the growing site and the orientation of the new spin are both determined by means of a pseudo-random number. Although the configuration energy of a MEM cluster, given by Eq.(1), resembles the Ising Hamiltonian, it should be noticed that the MEM is a nonequilibrium model in which new spins are 
continuously added, while older spins remain frozen and are not allowed to flip. The growth process naturally stops after the deposition of $N$ particles, when the network becomes completely filled.

Since finite-size effects for the MEM growing on small-world and BA scale-free networks are mild (see Refs. [33, 35] for detailed discussions), in this work we adopt a fixed network size, $N=10^{3}$, throughout. For any given set of defining parameters (i.e. the shortcut-adding probability $p$, the BA parameter $m$, the magnetic field $H$ and the temperature $T$ ), ensemble averages were calculated over $10^{4}$ different (randomly generated) networks, and considering typically 50 different (randomly chosen) seeds for each network configuration.

\section{Results and discussion}

\subsection{Advertising and opinion spreading in small-world networks}

The natural order parameter of a magnetic system is the total magnetization per site, i.e. 1

$$
M=\frac{1}{N} \sum S_{i}
$$

which, in the context of this work, is to be measured on the completely filled network. The normalized probability distribution of the magnetization, $P_{M}$, is shown in Figure 1 for different temperatures, shortcut densities and magnetic fields: (a) $p=0$ and $H=0.05$; (b) $p=0$ and $H=0.2$; (c) $p=0.5$ and $H=0.05$; (d) $p=0.5$ and $H=0.2$.

For the regular lattice (Figures 1(a)-(b)), the distributions undergo abrupt changes when passing from low to high temperatures. In the case of small fields (Figure 1(a)), the low-temperature distribution is dominated by sharp maxima taking place at $M= \pm 1$. Increasing temperature, these peaks retract while a local maximum develops near $M \approx 0.2$ forming a Gaussian-like distribution. Notice also that, due to the small but positive magnetic field, the distributions are skewed and biased towards positive values of the magnetization. For large fields (Figure 1(b)), the distributions are highly asymmetric and show the same kind of abrupt transition from a low-temperature sharp maximum at $M=1$ to high-temperature Gaussian-like shapes.

\footnotetext{
${ }^{1}$ Notice that, although this work is mainly motivated by social phenomena, a magnetic language is adopted throughout. Indeed, physical concepts such as temperature and magnetization, spin growth and clustering, ferromagnetic-paramagnetic phase transitions, etc, can be meaningfully re-interpreted in sociological/sociophysical contexts.
} 

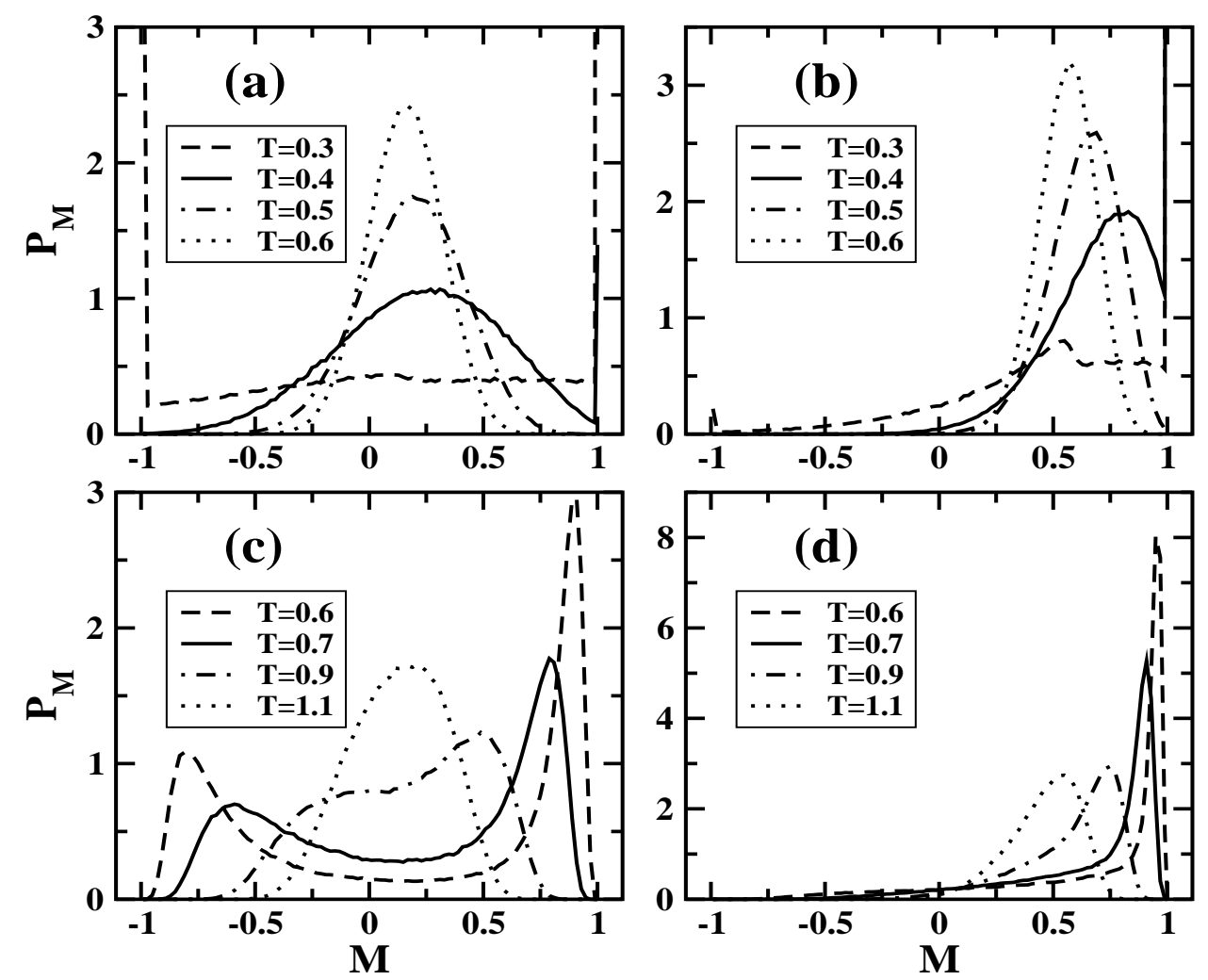

Figure 1: Normalized probability distributions of the magnetization for different temperatures, as indicated. The panels correspond to different shortcut densities and magnetic fields: (a) $p=0$ and $H=0.05$; (b) $p=0$ and $H=0.2$; (c) $p=0.5$ and $H=0.05$; (d) $p=0.5$ and $H=0.2$. Sharp maxima at $M= \pm 1$ have been truncated in panels (a)-(b).

In contrast, for the small-world network under small fields (Figure 1(c)) one observes the onset of two maxima located at $M=-M_{\text {neg }}$ and $M=M_{\text {pos }}$ (such that $0<M_{n e g}<M_{\text {pos }}<1$ ), which become sharper and approach $M= \pm 1$ as $T$ is gradually decreased. The smooth shift of the distribution maxima across $T \simeq T_{c}$, from $M \approx 0$ at high $T$ to the low-temperature nonzero spontaneous magnetizations $M=-M_{n e g}, M_{\text {pos }}$, is indeed the signature of true thermally-driven continuous phase transitions [38, 45]. Hence, the presence of shortcuts in the small-world network triggers a critical behavior in the irreversible growth of MEM clusters [33, analogously to phenomena observed in related equilibrium systems such as the Ising model [23, 24]. When strong fields are applied (Figure 1(d)), the negative maxima at $M=-M_{n e g}$ vanish, thus leading to highly asymmetric distributions peaked at $M=M_{\text {pos }}$ that tend to $M_{\text {pos }} \simeq 1$ as $T$ decreases. 


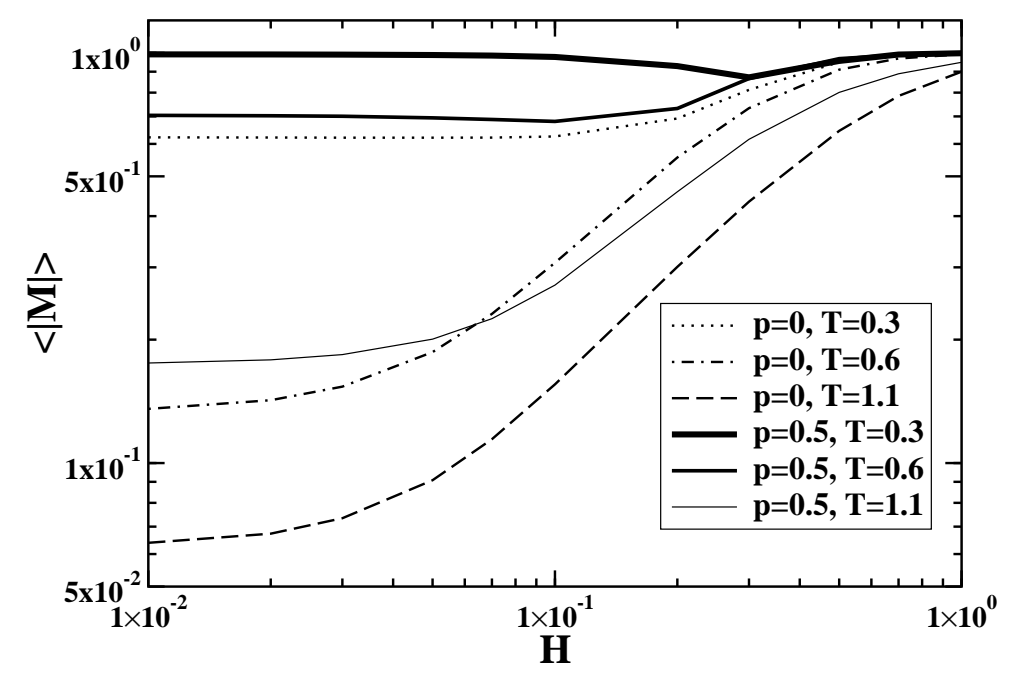

Figure 2: Ensemble-averaged absolute magnetization as a function of the magnetic field for different shortcut densities and temperatures, as indicated.

Since, for $H \ll 1$, the low-temperature probability distributions of the magnetization are nearly symmetric and sharply peaked around $M \approx \pm 1$, the ensemble-averaged total magnetization vanishes. This well-known shortcoming due to finite-size effects is avoided by considering the absolute value of the magnetization, $|M|$, as the actual order parameter [45, 46].

Figure 2 shows the ensemble-averaged absolute magnetization as a function of the applied magnetic field for different shortcut densities and temperatures, as indicated. For any value of the temperature, the order parameter plot for the small-world network (solid lines) lies above the corresponding one for the lattice (dotted/dashed lines), since shortcuts favor long-range ordering connections between distant clusters across the network. Moreover, as expected, most of the plots are monotonically increasing due to the ordering effect of the external field. Interestingly, however, a slight decrease in the absolute magnetization is observed at intermediate fields, $H=0.1-0.3$, for $p=0.5$ and $T=0.3$. This behavior is indeed due to the competition between the strong clustering tendency of neighboring spins at low temperatures, which favors the formation of extended parallel-oriented up and down domains, and the external magnetic field.

Figure 3 shows $p$-dependent, order-disorder transition curves on a $H$ vs $T$ phase diagram, where transition states are defined by the condition $\langle|M|\rangle=0.5$. Due to the long-range ordering effect of shortcut connections, transitions for small-world networks with higher shortcut densities are shifted towards higher temperatures. Moreover, due to the global reach of the ex- 


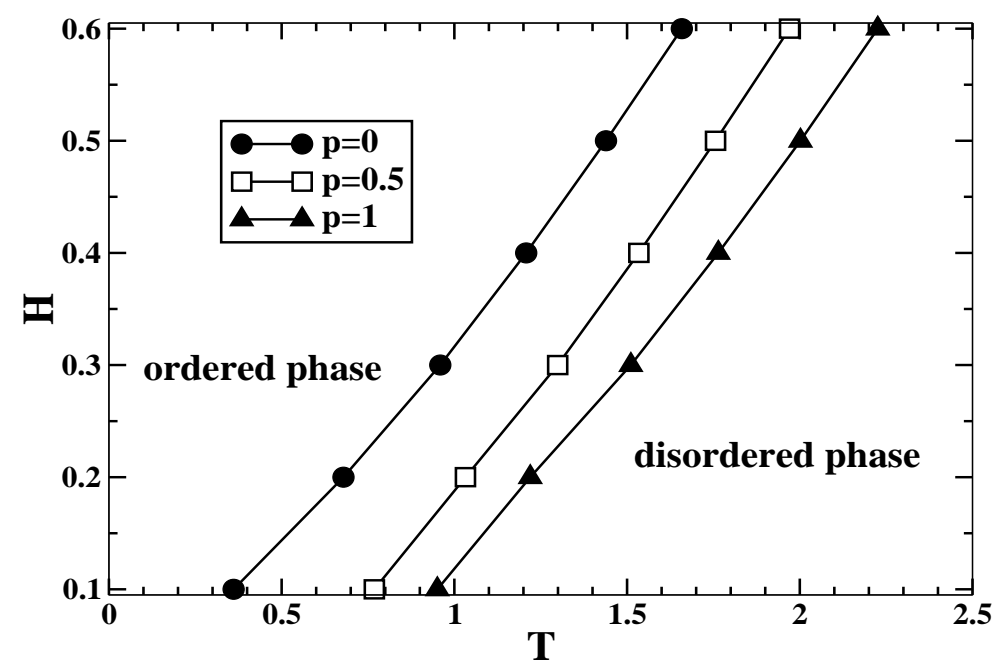

Figure 3: Phase diagram in the $H$ vs $T$ plane for different values of the shortcut fraction $p$, as indicated.

ternal field, the ordered phase is attainable even for very high temperatures. According to the sociophysical interpretation of this model, we conclude that consensus can be reached irrespective of the degree of intrinsic cohesion and interconnectedness (as given by the parameters $T$ and $p$, respectively) as long as a sufficiently intense, global mass media advertising campaign is carried out.

Figure 4 shows the transition temperature as a function of the shortcut fraction $p$ for different values of the external field, as indicated. The monotonic shift towards higher temperatures for small-world networks with higher connectivities is observed to be roughly independent of the external field. In order to exploit this approximate scaling property, we define an effective, shortcut-induced temperature shift $\Delta T_{\text {eff }}(p)=-\alpha p$ and re-plot the phase diagram in terms of $H$ vs $T_{\text {eff }} \equiv T+\Delta T_{\text {eff }}$, which is shown in the inset to Figure 4. Using $\alpha=0.45$, the small-world network data tend to collapse into a curve that can be well fitted by the relation $H=0.27 \times T_{\text {eff }}^{1.48}$ (solid line). As expected, lattice data show a distinct behavior: the $p=0$ transition is well fitted by $H=0.32 \times T_{e f f}^{1.23}$ (dashed line), which clearly departs from the small-world network data collapse.

In order to gain further insight into the dynamical features of the opinion spreading process, let us consider the effects of advertising campaigns with variable duration. For the sake of simplicity, we assume a constant deposition (or spreading) rate and measure time as $t \equiv n / N$, i.e. the fraction of already deposited particles, $n$, relative to $N$, the system's final size. At every stage 


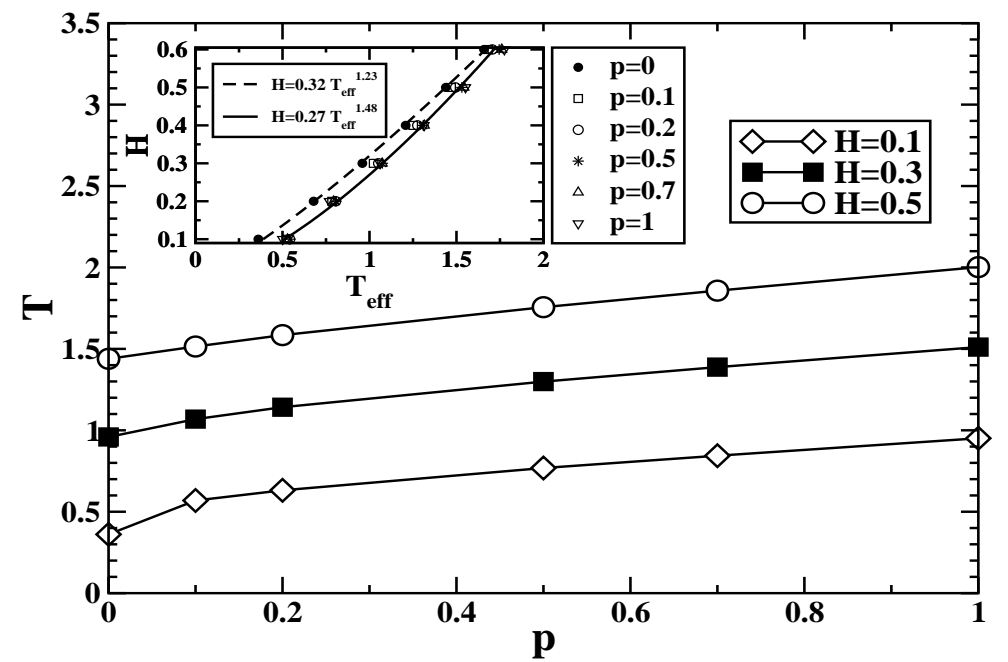

Figure 4: Order-disorder transition temperature as a function of the shortcut fraction $p$ for different values of the external field, as indicated. The inset shows the phase diagram $H$ vs $T_{\text {eff }}$, where the effective temperature $T_{\text {eff }}=$ $T-0.45 \times p$ leads to the collapse of the small-world network data. Separate fits to the lattice (dashed line) and small-world network (solid line) data are also shown.

during the growth process, we determine whether a given configuration has a majority of up spins $\left(n_{u p}>n / 2\right)$ and, after ensemble-averaging over many configurations, we measure the fraction of states with a majority of up spins, $f_{\text {up }}$. Since the seeds are chosen at random, the initial condition is $f_{u p}=0.5$ and the success of the campaign can be measured by the extent to which $f_{u p}$ approaches unity. We assume that the field is applied from $t=0$ to a specified time $t=t_{H}$; afterwards, the field is switched off. Hence, the parameter $0<t_{H} \leq 1$ represents the duration of the advertising campaign.

Figure 5 shows time profiles of $f_{u p}$ for small-world networks with $p=0.5$, different temperatures and magnetic fields: (a) $T=0.5$ and $H=0.1$; (b) $T=0.5$ and $H=0.5$; (c) $T=2$ and $H=0.1$; (d) $T=2$ and $H=0.5$. Different field switch-off times correspond to different plots on each panel, as indicated. Figure 5(a) shows that small fields are not capable of affecting the strong clustering tendency typical of low temperatures. Indeed, the magnetization probability distributions are sharply peaked at $M_{\text {neg }} \approx M_{\text {pos }} \approx 1$ (similarly to the low-temperature plot in Figure 1(c)) and are little influenced by the small external field even in the case of $t_{H}=1$. For low temperatures and strong fields, instead, $f_{u p}$ grows steadily with $t_{H}$ (Figure $5(\mathrm{~b})$ ), since strong fields deplete the probability distribution for negative values of the 

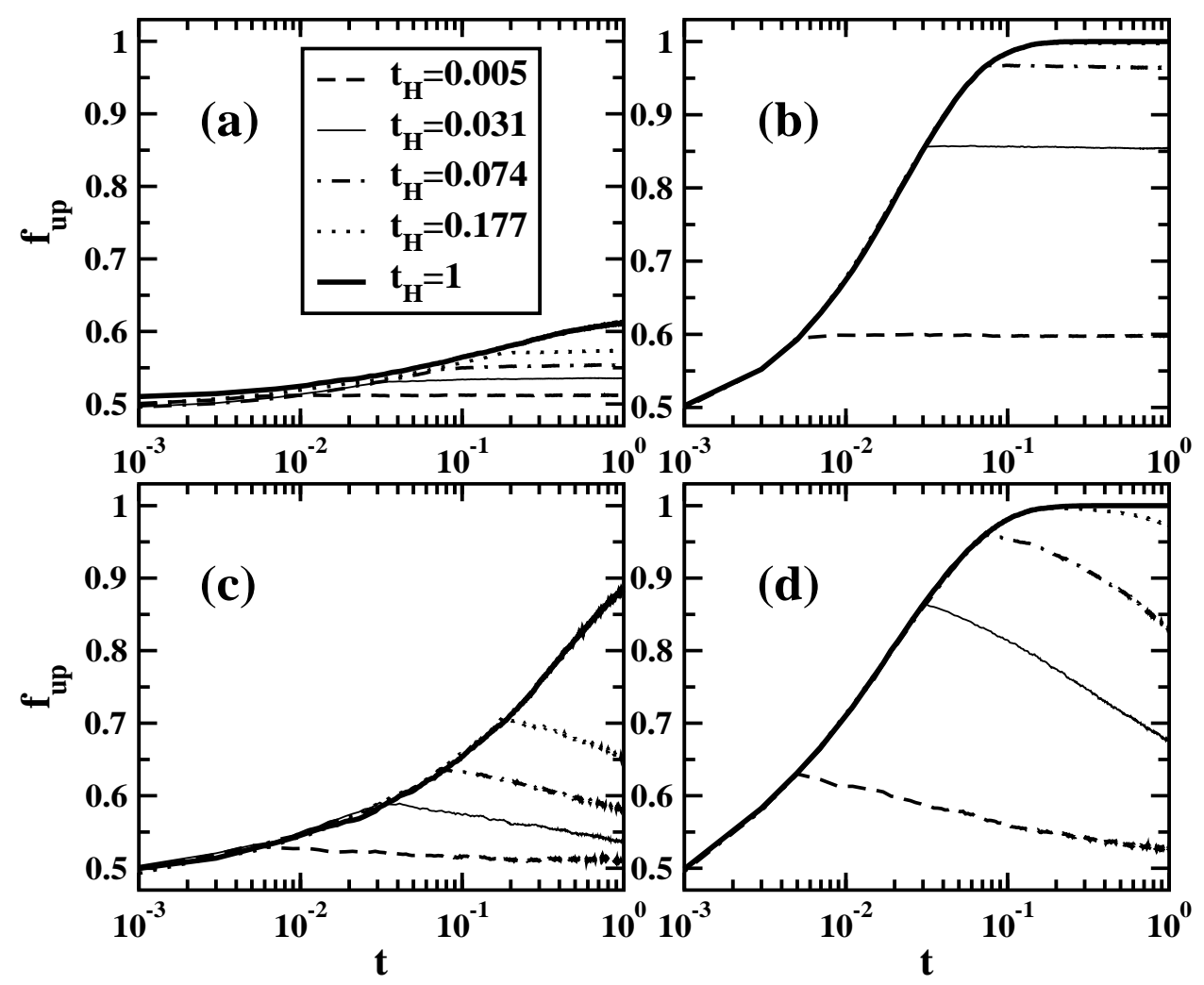

Figure 5: Time profiles for the fraction of states with a majority of up spins, $f_{u p}$, for small-world networks with $p=0.5$ and different field switch-off times, $t_{H}$, as indicated. The panels correspond to different temperatures and magnetic fields: (a) $T=0.5$ and $H=0.1$; (b) $T=0.5$ and $H=0.5$; (c) $T=2$ and $H=0.1$; (d) $T=2$ and $H=0.5$.

magnetization (recall Figure 1(d)). Moreover, $f_{\text {up }}$ remains constant after the field is switched off due to the lack of sizable thermal fluctuations. Notice that highly successful final results (i.e. $f_{u p} \sim 1$ for $t \rightarrow 1$ ) can be achieved with intense but very short campaigns $\left(t_{H}<0.1\right)$. Figure $5(\mathrm{c})$ shows that, for high temperatures and small fields, $f_{u p}$ strongly depends on the campaign duration: $f_{u p}$ increases monotonically with $t_{H}$ and is close to unity for $t_{H}=1$. Comparing to Figure 5(a), one observes that higher temperatures increase the overall response sensitivity of the system under the influence of small fields. The case of high temperatures and large fields is shown in Figure $5(\mathrm{~d})$. The strong fields naturally drive the system towards large values of $f_{\text {up }}$; however, whenever the fields are switched off, the trend towards thermal disorder sets back on and $f_{u p}$ decreases again.

According to these results, the best cost-effective strategy to achieve a de- 


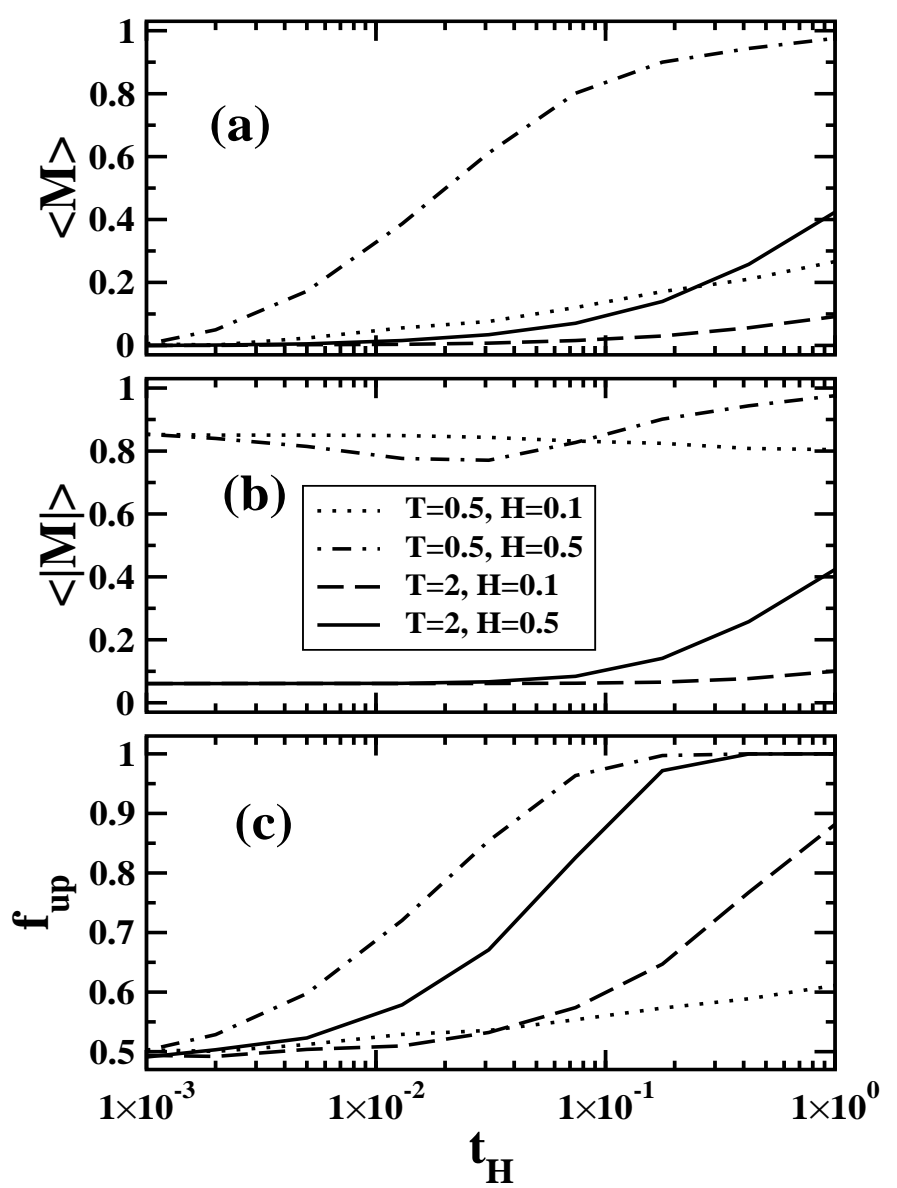

Figure 6: Dependence of the ensemble-averaged final states $(t=1)$ on the field switch-off time, $t_{H}$, for different temperatures, external fields and observables: (a) the total magnetization, (b) the absolute magnetization, and (c) the fraction of final states with a majority of up spins. These results are for small-world networks with $p=0.5$.

sired majority consensus $\left(f_{u p} \rightarrow 1\right.$ for $t \rightarrow 1$ ) depends crucially on the degree of intrinsic cohesion tendency of the society, here modeled by the temperature. In the case of strongly cohesive social groups (low $T$ ), intense, short campaigns yield larger consensus levels than mild, long ones. Interestingly, however, the opposite is true in the scenario of weakly cohesive societies (high $T \longdiv { 2 }$.

\footnotetext{
${ }^{2}$ Notice that the low- and high-temperature regions are relative to the corresponding zero-field critical temperature. For SWNs with $N=10^{3}$ and $p=0.5$, the zero-field transition is located at $T_{c} \approx 0.6$ 33. Also recall that temperatures are measured in units of the nearest-neighbor coupling constant throughout (see Sect. 2 for details).
} 


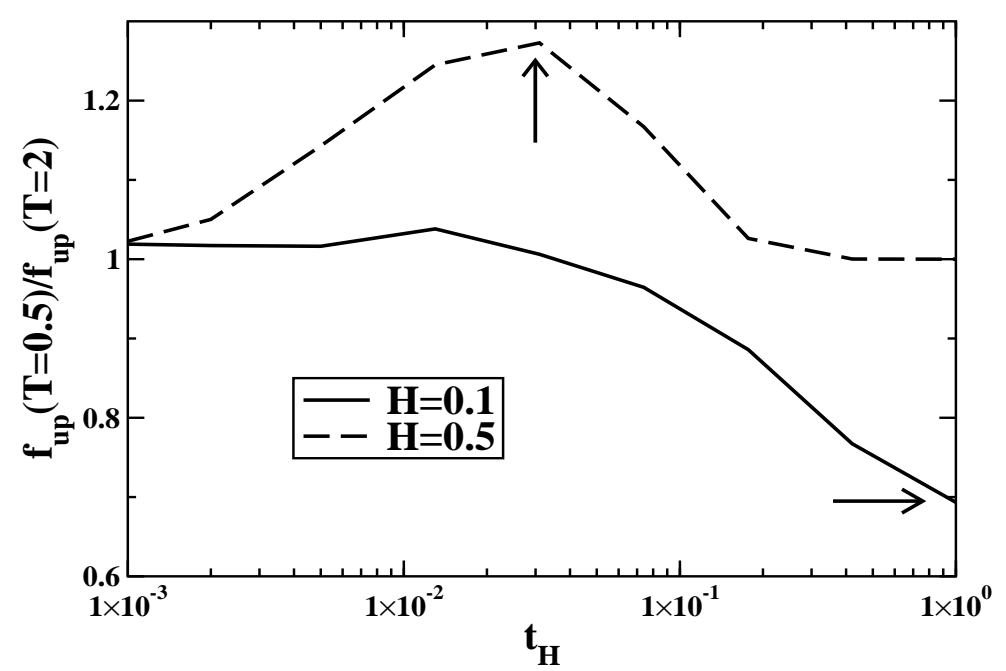

Figure 7: Ratio $f_{u p}(T=0.5) / f_{u p}(T=2)$ as a function of the field switch-off time $t_{H}$ for small-world networks with $p=0.5$ under different external fields, as indicated.

Figure 6 shows the dependence of the ensemble-averaged final states (i.e. $t=1$ ) on the campaign duration (or, equivalently, the field switch-off time, $\left.t_{H}\right)$ for different temperatures, external fields and observables: (a) the total magnetization, (b) the absolute magnetization, and (c) the fraction of final states with a majority of up spins. The results shown correspond to smallworld networks with shortcut fraction $p=0.5$.

For low temperatures and strong fields (dash-dotted line plots), the system becomes rapidly ordered in the up direction and stays in the up-ordered state even after the field is withdrawn. Hence, $\langle M\rangle$ and $f_{u p}$ follow a similar pattern, namely a monotonic, steadily increasing behavior as $t_{H}$ is increased. The absolute magnetization is close to unity but displays a dip, which is due to the passing of the down-ordered probability distribution, through the zero-magnetization region, towards the up-ordered side. This behavior is similar to the dip previously observed in the thick solid line plot of Figure 2. For low temperatures and small fields (dotted lines), the positive fields are not capable of significantly affecting the strongly clustered down-ordered domains, thus leading to small but moderately increasing values for $\langle M\rangle$ and $f_{u p}$. In contrast, $\langle|M|\rangle$ is large but slightly decreasing, as explained by mild shifting effects of the magnetization probability distribution towards the positive side. When high temperatures are considered (long-dashed and solid lines), the magnetization probability distributions are nearly Gaussian with positive mean values. Hence, $\langle M\rangle$ and $\langle|M|\rangle$ are expected to be similar and 


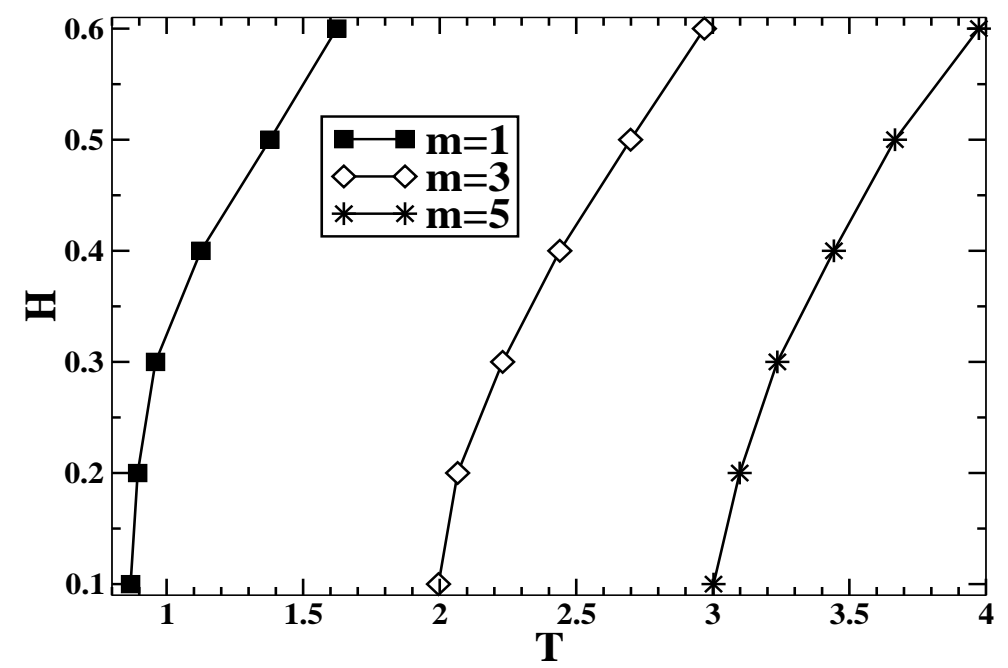

Figure 8: Phase diagram in the $H$ vs $T$ plane for different values of the Barabási-Albert parameter $m$, as indicated.

generally small. However, since in the $H \rightarrow 0$ limit these distributions are Gaussians centered at $M=0$, even slight shifts towards $M>0$ when small fields are applied lead ultimately to fairly large values of $f_{u p}$. This explains the fact that, for high temperatures, mild, long campaigns yield higher $f_{u p}$ values than short, intense ones.

Figure 7 shows the ratio $f_{u p}(T=0.5) / f_{u p}(T=2)$ as a function of $t_{H}$ for different fields. For $H=0.1$, the ratio remains fairly constant about unity up to $t_{H} \simeq 3 \times 10^{-2}$, while it decreases monotonically for higher values of $t_{H}$ and reaches a minimum at $t_{H}=1$ (horizontal arrow), where the hightemperature campaign is more successful by more than $40 \%$ relative to the low-temperature one. Hence, mild, long campaigns are optimal for (hightemperature) weakly cohesive societies. For $H=0.5$, the ratio peaks at $t_{H}=$ $3 \times 10^{-2}$ (vertical arrow) and then decreases towards unity for larger values of $t_{H}$, thus showing that for (low-temperature) highly cohesive societies, short, intense campaigns are the best strategy.

\subsection{Advertising and opinion spreading in scale-free net- works}

Unlike the case of small-world networks, the highly inhomogeneous degree distribution of scale-free networks represents a scenario in which social roles differ significantly among individuals. In particular, hubs in the scale-free network represent highly influential social leaders, whose opinions can po- 


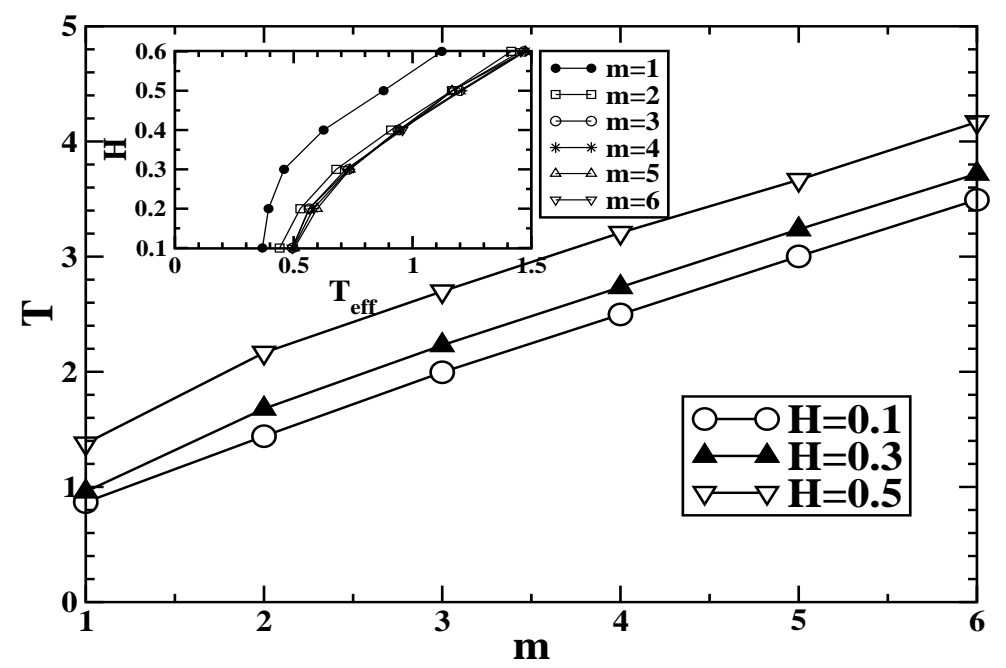

Figure 9: Order-disorder transition temperature as a function of the Barabási-Albert parameter $m$ for different values of the external field, as indicated. The inset shows the phase diagram $H$ vs $T_{\text {eff }}$, where the effective temperature $T_{\text {eff }}=T-0.50 \times m$ leads to an approximate data collapse for scale-free networks with $m>1$.

tentially affect the decisions of many other individuals in the society.

The paradigmatic type of scale-free network is based on the preferential attachment growth mechanism: a new node with $m$ edges is added at every time step and is connected to $m$ different nodes already present in the system, where the probability for an already existing node to acquire a new link is proportional to its degree. This simple rule leads to an ensemble of BarabásiAlbert (BA) scale-free networks, which are characterized by power-law degree distributions with exponent $\gamma=3$.

Figure 8 shows order-disorder transition plots on a $H$ vs $T$ phase diagram for the MEM growing on SF networks with different values of the BA parameter $m$. Since the overall average degree grows linearly with $m$, the transition curves for SF networks with higher link densities are shifted towards higher temperatures. Moreover, due to the global reach of the external field, the ordered phase is attainable even for very high temperatures. Comparing to the SWN results (see Figure 3), we observe that, irrespective of the details of the underlying complex network substrate, consensus and order can be reached for any level of intrinsic cohesion and interconnectedness.

Figure 9 shows the transition temperature as a function of the BA parameter $m$ for different values of the external field, as indicated. Except for the $m=1$ case, the monotonic shift towards higher temperatures appears 


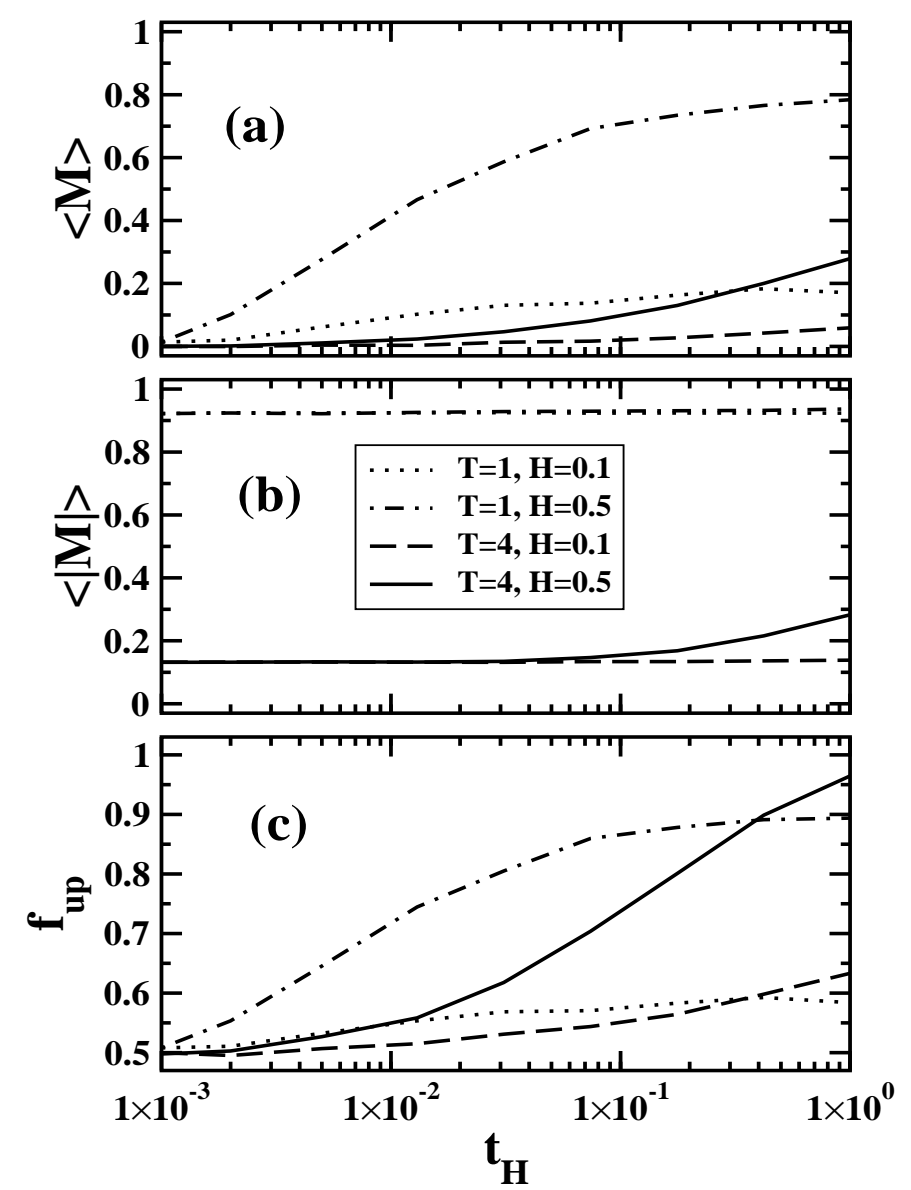

Figure 10: Dependence of the ensemble-averaged final states $(t=1)$ on the field switch-off time, $t_{H}$, for different temperatures, external fields and observables: (a) the total magnetization, (b) the absolute magnetization, and (c) the fraction of final states with a majority of up spins. These results are for scale-free networks with BA parameter $m=3$.

to be roughly independent of the external field, which suggests to define an effective, $m$-induced temperature shift $\Delta T_{\text {eff }}(m)=-\alpha m$. Using $\alpha=0.5$, we obtain an approximate data collapse for scale-free networks with $m>1$, as shown in the inset to Figure 8. Comparing to the small-world network data shown in Figure 4, we confirm that, from a qualitative point of view, the critical behavior induced by random connections is generally characteristic of complex network substrates.

Figure 10 shows the dependence of the ensemble-averaged final states as a function of the field switch-off time, $t_{H}$, for different temperatures 3 ,

\footnotetext{
${ }^{3}$ Since, for BA-SF networks with $N=10^{3}$ and $m=3$, the zero-field transition is located
} 


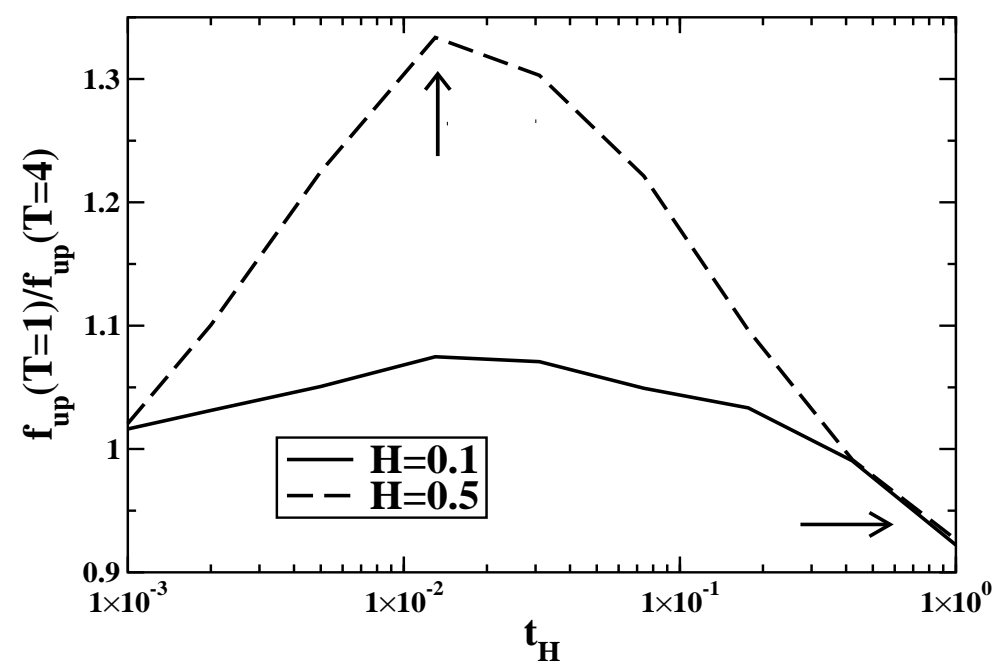

Figure 11: Ratio $f_{u p}(T=1) / f_{u p}(T=4)$ as a function of the field switch-off time $t_{H}$ for scale-free networks with BA parameter $m=3$ under different external fields, as indicated.

external fields and observables: (a) the total magnetization, (b) the absolute magnetization, and (c) the fraction of final states with a majority of up spins. The results shown correspond to scale-free networks with BA parameter $m=$ 3. From a qualitative point of view, these results compare well to the smallworld data shown above (see Figure 6 and discussion).

Finally, let us consider in Figure 11 the ratio $f_{u p}(T=1) / f_{u p}(T=4)$ as a function of $t_{H}$ for different fields, focusing again on scale-free networks with BA parameter $m=3$. For $H=0.1$, the ratio shows a slight increase for small values of $t_{H}$ up to about $t_{H} \simeq 3 \times 10^{-2}$, while it decreases monotonically for higher values of $t_{H}$ and reaches a minimum at $t_{H}=1$ (horizontal arrow). This behavior resembles the observations made above for SWNs (recall Figure 7 ), although the effect apparent here is milder. For $H=0.5$, the ratio peaks at $t_{H} \approx 1.5 \times 10^{-2}$ (vertical arrow) and then decreases for larger values of $t_{H}$. This confirms that, irrespective of the topological details of the complex network substrate, short, intense campaigns are the best strategy to successfully convey a message across a highly cohesive society. Incidentally, notice also that, unlike the SWN case, the $t_{H} \rightarrow 1$ tail seems to be roughly independent of the external field.

at $T_{c} \approx 2\left[35\right.$, the chosen temperatures roughly correspond to $T=0.5 T_{c}$ and $T=2 T_{c}$, respectively. 


\section{Conclusions and Outlook}

In order to investigate advertising and irreversible opinion spreading phenomena, we studied the magnetic Eden model (MEM) growing on small-world and scale-free networks with externally applied magnetic fields. In this nonequilibrium model, the opinion or decision of an individual is affected by those of their acquaintances, but opinion changes (analogous to spin flips in an Ising-like model) do not occur.

The model studied in this work could be realistically applied to sociological scenarios in which individuals are subject to highly polarized, short term, binary choice situations under the influence of advertising/marketing mass media campaigns. Given these conditions, opinions are not expected to fluctuate and "thermalize". Some examples are binary voting situations, such as a ballotage or referendum, and market competition scenarios between two leading products, where the validity of this model could be empirically tested using polls, surveys and/or sales performance data.

The interplay and competition between the intrinsic local disorder and the externally applied ordering field was investigated by means of orderparameter probability distributions and ensemble averages, leading to a temperature vs. magnetic field order-disorder phase diagram. The small-world network's shortcut fraction was found to shift the effective temperature of the system, suggesting a simple scaling relation that allowed a collapse of all small-world network data. Later, the effects of advertising campaigns with variable duration was discussed. The best cost-effective strategy to achieve a desired majority consensus was found to depend crucially on the intrinsic cohesion tendency of the society. In the scenario of strongly cohesive social groups, intense, short campaigns are observed to be more successful than mild, long ones. However, the opposite conclusion holds in the case of weakly cohesive societies.

The observed phenomena, first presented and discussed for small-world network substrates, were compared to corresponding results obtained using scale-free network geometries. We found that, irrespective of the details of the underlying complex network topology, consensus and order can be reached for any level of intrinsic cohesion and interconnectedness. Moreover, the effects of advertising campaigns with variable duration, as well as our conclusions regarding optimal strategies to achieve a majority consensus, remain valid irrespective of the specific type of complex network substrate.

The magnetic Eden model, being different in nature from related equilibrium spin systems such as the Ising model, can certainly provide valuable, complementary insight into dynamical and critical aspects of the spreading of opinions in a society. Since a characteristic feature of the magnetic Eden 
model is the absence of thermalization, it may be interesting to study a hybrid model with an external parameter controlling the rate of thermalization relative to the rate of growth. This generalized model could be tuned from pure MEM-like growth to pure Ising-like thermalization, which might be an appropriate representation of sociological scenarios where the opinion of individuals is neither completely frozen nor fully thermalized.

Hopefully, the present findings will thus contribute to the growing interdisciplinary efforts in the fields of sociophysics, complex networks, and nonequilibrium statistical physics, and stimulate further work.

\section{Acknowledgments}

This work was supported by the James S. McDonnell Foundation and the National Science Foundation ITR DMR-0426737 and CNS-0540348 within the DDDAS program, as well as by CONICET, ANPCyT, and UNLP (Argentina).

\section{References}

[1] S. Galam, Y. Gefen, Y. Shapir, Math. J. Soc. 9, 1 (1982).

[2] S. M. de Oliveira, P. M. C. de Oliveira, D. Stauffer, Non-Traditional Applications of Computational Statistical Physics (B.G. Teubner, Stuttgart, 1999)

[3] W. Weidlich, Sociodynamics: a Systematic Approach to Mathematical Modelling in the Social Sciences (Harwood Academic Publishers, Amsterdam, 2000)

[4] D. Stauffer, S.M. de Oliveira, P.M.C. de Oliveira, J.S. Sa Martins, Biology, Sociology, Geology by Computational Physicists (Elsevier, Amsterdam, 2006)

[5] C. Castellano, S. Fortunato, V. Loreto, arXiv:0710.3256 (to appear in Rev. Mod. Phys.).

[6] F. Schweitzer, J. A. Hołyst, Eur. Phys. J. B 15, 723 (2000)

[7] K. Sznajd-Weron, J. Sznajd, Int. J. Mod. Phys. C 11, 1157 (2000)

[8] S. Galam, Eur. Phys. J. B 25, 403 (2002) 
[9] M. Kuperman, D. Zanette, Eur. Phys. J. B 26, 387 (2002)

[10] D. Vilone, A. Vespignani, C. Castellano, Eur. Phys. J. B 30, 399 (2002)

[11] C. Schulze, Int. J. Mod. Phys. C 14, 95 (2003)

[12] C. J. Tessone, R. Toral, P. Amengual, H. S. Wio, M. San Miguel, Eur. Phys. J. B 39, 535 (2004)

[13] S. Galam, Europhys. Lett. 70, 705 (2005).

[14] J.C. González-Avella, M.G. Cosenza, K. Tucci, Phys. Rev. E 72, 065102 (2005).

[15] J.C. González-Avella, V.M. Eguíluz, M.G. Cosenza, K. Klemm, J.L. Herrera, M. San Miguel, Phys. Rev. E 73, 046119 (2006).

[16] M. S. de la Lama, I. G. Szendro, J. R. Iglesias, H. S. Wio, Eur. Phys. J. B 51, 435 (2006)

[17] C.M. Bordogna, E.V. Albano, J. Phys.: Condens. Matter 19, 065144 (2007)

[18] K. Mazzitello, J. Candia, V. Dossetti, Int. J. Mod. Phys. C 18, 1475 (2007)

[19] J.C. González-Avella, M.G. Cosenza, K. Klemm, V.M. Eguíluz, M. San Miguel, J. Art. Soc. Soc. Simul. 10, 9 (2007).

[20] J. Candia, K. Mazzitello, J. Stat. Mech. P07007 (2008)

[21] S. Galam, Int. J. Mod. Phys. C 19, 409 (2008).

[22] S. Galam, Physica A 238, 66 (1997).

[23] A. Barrat, M. Weigt, Eur. Phys. J. B 13, 547 (2000)

[24] M. Gitterman, J. Phys. A: Math. Gen. 33, 8373 (2000)

[25] A. Aleksiejuk, J. A. Hołyst, D. Stauffer, Physica A 310, 260 (2002)

[26] P. Svenson, D.A. Johnston, Phys. Rev. E 65, 036105 (2002)

[27] C.P. Herrero, Phys. Rev. E 65, 066110 (2002)

[28] K. Medvedyeva, P. Holme, P. Minnhagen, B.J. Kim, Phys. Rev. E 67, 036118 (2003) 
[29] C.P. Herrero, Phys. Rev. E 69, 067109 (2004)

[30] S. Milgram, Psychol. Today 2, 60 (1967)

[31] D.J. Watts, Small Worlds (Princeton University Press, Princeton, 1999)

[32] D.J. Watts, S.H. Strogatz, Nature (London) 393, 440 (1998)

[33] J. Candia, Phys. Rev. E 74, 031101 (2006)

[34] J. Candia, J. Stat. Mech. P09001 (2007)

[35] J. Candia, Phys. Rev. E 75, 026110 (2007)

[36] M. Ausloos, N. Vandewalle, R. Cloots, Europhys. Lett. 24, 629 (1993)

[37] J. Candia, E.V. Albano, Eur. Phys. J. B 16, 531 (2000)

[38] J. Candia, E.V. Albano, Phys. Rev. E 63, 066127 (2001)

[39] J. Candia, E.V. Albano, Int. J. Mod. Phys. C 19, 1617 (2008)

[40] B. Bollobás, F.R.K. Chung, SIAM J. Discrete Math. 1, 328 (1988)

[41] M.E.J. Newman, D.J. Watts, Phys. Rev. E 60, 7332 (1999)

[42] A.-L. Barabási and R. Albert, Science 286, 509 (1999).

[43] R. Albert and A.-L. Barabási, Rev. Mod. Phys. 74, 47 (2002).

[44] S. N. Dorogovtsev and J. F. F. Mendes, Evolution of Networks (Oxford University Press, New York, 2003).

[45] K. Binder, D.W. Heermann, Monte Carlo simulation in statistical physics: an introduction, 4th ed. (Springer-Verlag, Berlin, 2002)

[46] D.P. Landau, K. Binder, A guide to Monte Carlo simulations in Statistical Physics (Cambridge University Press, Cambridge, 2000) 